\title{
Características mecánicas de los agentes adhesivos en dientes con dentinogenesis imperfecta
}

DOI: $10.46932 / \mathrm{sfjdv2n2-173}$

Received in: March 1st, 2021

Accepted in: May 30th, 2021

\section{DDs MSc Jorge Eliecer Patiño Giraldo}

Institution I,II,II,IV Universidad Antonio Nariño, Colombia

Av. Bolívar \#49 Norte -30 Armenia (Quindío), Colombia. 057-6-7494981

E-mail: cirujanojunior@gmail.com

DDs Camilo Andrés Agudelo Marín

Institution I,II,II,IV Universidad Antonio Nariño, Colombia.

Av. Bolívar \#49 Norte -30 Armenia (Quindío), Colombia. 057-6-7494981

\section{DDs Juan David Ospina Sanmigue}

Institution I,II,II,IV Universidad Antonio Nariño, Colombia.

Av. Bolívar \#49 Norte -30 Armenia (Quindío), Colombia. 057-6-7494981

DDs José Julián Ramírez Torres

Institution I,II,II,IV Universidad Antonio Nariño, Colombia. Av. Bolívar \#49 Norte -30 Armenia (Quindío), Colombia. 057-6-7494981

\section{RESUMEN}

Introducción: El desarrollo de la odontología conservadora ha generado el surgimiento y evolución de los sistemas adhesivos; los procesos de adhesión dependen del material, la experiencia del operador, las características del tejido y del control de las condiciones locales. La dentinogenesis imperfecta (DI) es una condición del desarrollo del diente que afecta el tejido dentinario, en la cual se ven comprometidas tanto la dentición temporal como permanente. Objetivos: Evaluar el comportamiento mecánico de los sistemas adhesivos en dientes sanos y dientes con DI. Materiales y métodos: Se realizaron 10 pruebas de microtensión en dientes sanos y 10 pruebas de microtensión en dientes con DI; Se verificó la distribución normal de los datos con la prueba Kolmororov - Smirnof, Se utilizó la prueba t student para analizar las diferencias entre los grupos con respecto a los valores de microtensión, además se utilizó la prueba taub de Kendall para establecer correlación entre dientes y tipo de falla. Resultados: Los dientes con dentinogenesis imperfecta presentan un grado de adhesión significativamente menor con respecto a los dientes sanos. Conclusión: Los dientes con DI presentan fallas en la adhesión debido a la estructura macro y microscópica, sin embargo, presentaron cierto grado de adhesión.

Palabras clave: Dentinogenesis imperfecta, adhesión dental, sistemas adhesivos, adhesión en dentina, microtensión.

\section{ABSTRACT}

Introduction: The development of conservative dentistry has generated the emergence and evolution of adhesive systems; The adhesion processes depend on the material, operator experience, tissue characteristics and control of local conditions. Imperfect dentinogenesis (DI) is a tooth development condition that affects dentinal tissue, in which both temporary and permanent dentition are compromised. Objectives: To evaluate the mechanical behavior of adhesive systems in healthy teeth and teeth with imperfect dentinogenesis. Materials and methods: 10 microtensión tests were performed on healthy teeth 
and 10 microtensión tests on teeth with imperfect dentinogenesis; The normal distribution of the data was verified with the Kolmororov - Smirnof test. The t-student test was used to analyze the differences between the groups with respect to microtensión values, in addition the Taub Kendall test was used to establish correlation between teeth and Type of fault. Results: Teeth with DI have a significantly lower degree of adhesion with respect to healthy teeth. Conclusion: Teeth with DI have adhesion failures due to the macro and microscopic structure. however, they presented a degree of adhesion.

Keywords: imperfect dentinogenesis, dental adhesion, adhesive systems, dentin adhesion, microtensión.

\section{INTRODUCCIÓN}

El desarrollo de la odontología conservadora ha generado el surgimiento y evolución de los sistemas adhesivos $^{(1)}$, es importante conocer que para el éxito de este tipo de restauraciones se debe tener en cuenta diversos factores que deben ser controlados debido a la sensibilidad de las técnicas. ${ }^{(2)}$ Por lo tanto, los procesos de adhesión no dependen únicamente del material, sino también de la experiencia del operador, la cantidad y tipo de tejido y del control de las condiciones locales. Dependiendo del tipo de tejido en el cual se realice la técnica adhesiva el procedimiento y las características tienen algunas variaciones; la adhesión a dentina se logra debido a la formación de la capa híbrida, la cual está conformada por la dentina desmineralizada y la infiltración de un líquido orgánico, con capacidad de polimerizar entre las fibras colágenas. Parte del líquido también penetra en los túbulos dentinarios, formando los llamados "tags" de resina. Debido a la complejidad de su estructura, la adhesión a la dentina se ha considerado más difícil y menos predecible que la adhesión a esmalte. ${ }^{(2)}$

Algunas condiciones del desarrollo dental pueden afectar el proceso de adhesión, dentro de las más importantes tenemos la amelogénesis imperfecta, dentinogenesis imperfecta y displasia dentinaria. ${ }^{(3)}$

La DI es una condición del desarrollo del diente que afecta el tejido dentinario, en la cual se ven comprometidas tanto la dentición temporal como permanente. Se define según la literatura como tipo I, II y III. Actualmente se conoce que su prevalencia es de uno en 8000 personas. $^{(4)}$

\section{MATERIALES Y MÉTODOS}

Se realizó una investigación de tipo cuasi experimental in vitro, que tuvo como objetivo comparar las fuerzas de adhesión en dientes sanos y con dentinogenesis imperfecta tipo II. Para este estudio se realizó la recolección de un grupo de dientes con dentinogenesis imperfecta tipo II (indicados para exodoncia) y otro grupo de dientes sin alteración estructural en la clínica de adultos de la Universidad Antonio Nariño (UAN) sede Armenia Quindío Colombia, (se eligieron terceros molares en los cuales estaba indicada la exodoncia). Posteriormente a los dos grupos de dientes se les realizó limpieza mecánica del tejido blando a nivel radicular con curetas de Gracey retirando fibras gingivales, ligamento periodontal 
y tejidos blandos que estaban alrededor del diente. Las muestras recolectadas permanecieron en agua destilada desionizada en un refrigerador a una temperatura nominal de $4^{\circ} \mathrm{C}$ en un recipiente con selle hermético con tiempo de almacenamiento no superior a seis meses, hasta 24 horas antes de realizar las pruebas, según la recomendación de la norma técnica Colombiana 4882. ${ }^{(5)}$

Se colocaron los dientes en un soporte con godiva de baja fusión, el soporte se llevó a la mordaza abrasiva de la máquina de corte; Se realizó un corte mesodistal paralelo al plano oclusal a nivel de tercio medio coronal, con la máquina de corte de precisión IsoMet LS (BUHELER,Illinois) a una velocidad de 300 rpm, se utilizó una hoja de diamante para realizar los cortes, se utilizó irrigación de agua para evitar la desecación y el recalentamiento, verificando que únicamente quedará expuesta la dentina. ${ }^{(6)}$

La dentina expuesta se froto con lija de calibre delgado y posteriormente se aplicó ácido fosfórico al 35\%(Scotchbond, 3M) por 10 segundos, se enjuagó por 20 segundos y se secó con tiras de papel absorbente observando que la dentina no estuviera desecada, se aplicó la primer capa de agente adhesivo (Adper Scotchbond, 3M) y se frotó por 20 segundos, posteriormente se aplicó una segunda capa de adhesivo y se frotó por 20 segundos; se fotopolimerizó por 20 segundos, este procedimiento fue llevado a cabo por el mismo operador. ${ }^{(6)}$

Utilizando una espátula de teflón se realizaron cilindros de resina (Filtek z250, 3M), de 6 mm de diámetro y $5 \mathrm{~mm}$ de alto, mediante la técnica incremental, con incrementos de $1 \mathrm{~mm}$, para estandarizar área de adhesión. Luego de haber realizado la adhesión los especímenes se llevaron a una incubadora que permaneció a una temperatura de $37^{\circ} \mathrm{C}$ por 24 horas. ${ }^{(7)}$

Las muestras fueron llevadas a la máquina de corte ISOMET, y se realizaron cortes de incisal hacia apical, de mesial a distal y se repitió el procedimiento de vestibular a palatino, para obtener microbarras de $0.9 \mathrm{~mm} 2 .{ }^{(6)}$

Las microbarras se ubicaron en un portaobjetos a $5 \mathrm{~mm}$ de la interface diente-resina, luego fueron llevados al INSTRON 3345(INSTRON, Illinois donde se aplicó la carga a una velocidad de 1mm/min y los valores de microtensión se obtuvieron en Mpa. Se llevaron las muestras a un estereomicroscopío para determinar el tipo de falla producido, y se determinó cuantas fueron de adhesión, cohesión o cuantas fueron mixtas.

Los datos se ingresaron en una base de datos de Excel (Microsoft Office 2013) y se verificaron para evitar errores de ingreso. La unidad para el análisis estadístico básico fue la barra obtenida de los dientes. Todo el manejo de datos y pruebas estadísticas se realizó usando un paquete de software estadístico SPSS versión 24. Los promedios, las desviaciones estándar, de los dos grupos, serán calculados para la descripción de los datos. Se verificó la distribución normal de los datos con la prueba KolmogorovSmirnov figura1, Se utilizó la prueba t student para analizar las diferencias entre los grupos con respecto 
a los valores de microtensión, además se utilizó la prueba taub de Kendall para establecer correlación entre dientes y tipo de falla. La graficación se realizó con el PRISMA nivel de confiabilidad de 95\%.

\section{RESULTADOS}

En este estudio se evaluaron 20 muestras divididas en dos grupos (10 con dentinogenesis imperfecta y 10 sanos), los dientes obtenidos fueron molares de pacientes que asistieron a la clínica de la universidad Antonio Nariño. A la totalidad de las muestras se le aplicaron pruebas de microtensión y evaluación de tipo de falla, así como pruebas para verificar la distribución normal de los datos obtenidos.

El promedio de microtensión en megapascales del grupo de dientes sanos fue de 38,02 con una desviación estándar de 4,6 y el promedio en el grupo de dientes con dentinogenesis imperfecta fue de 20,07 y una desviación estándar de 4,1. Se encontraron diferencias significativas entre los dos grupos en cuanto a los valores de microtensión. El grupo con las muestras de dentinogenesis imperfecta presento valores de fuerza de adhesión significativamente menores con respecto al grupo de dientes sanos figura 2.

En cuanto al tipo de falla que presentaron las muestras, la más frecuente fue la falla adhesiva (45\%), se infiere entonces que las fallas se producen en mayor porcentaje en la interface adhesivo-dentina, La tabla 1 muestra la distribución por frecuencias del tipo de fallas.

Se evaluó si existía relación entre los grupos estudiados y el tipo de falla, se encontró una relación estadísticamente significativa entre el grupo con dentinogenesis imperfecta y la falla adhesiva tabla 2. También se encontró diferencia significativa $(\mathrm{p}<0,05)$ en la fuerza de adhesión dependiendo del tipo de falla (adhesiva y cohesiva).

\section{DISCUSIÓN}

Para las pruebas de microtensión aplicadas en dientes sanos se obtuvo una media de 38.02 Mpa, valor que concuerda con lo reportado por Seow y cols, ${ }^{(3)}$ quienes encontraron valores de resistencia adhesiva comprendidas dentro del rango de 55 Mpa y 38 Mpa. Los valores de microtensión obtenidos del meta análisis realizado por Munk y cols donde se evaluaron 298 artículos de estudios comprendidos entre 2002 y 2010, muestran valores de fuerzas adhesivas que se pueden comparar con ambos grupos para diferentes adhesivos. ${ }^{(8)}$ Ahora bien los dientes con dentinogenesis imperfecta del presente estudio reportaron valores similares al sistema Adper Prompt L-pop (3M ESPE )(1 paso) y superior a One Pop Bond F (Tokuyama)(1 paso); para dientes normales este estudio muestra valores de fuerzas de adhesión similares al sistema Xeeno III(Dentsply)(1 paso) y superiores Clearfill SE (Kuraray)(2 paso), Adper Scotchbond(3M ESPE)(3 pasos), One Step (Bisco)(2 pasos). ${ }^{(8)}$ 
El presente estudio mostró que los valores de microtensión para dientes sanos se encuentran cercanos a $38.02 \mathrm{MPa}( \pm 4.6)$ y para dentinogenesis imperfecta se encontraron valores cercanos a 20.07 $\mathrm{MPa}( \pm 4.1)$ observando una diferencia estadísticamente significativa en la fuerzas de adhesión entre los dos tipos de dentina, esta diferencia se puede explicar teniendo en cuenta dos factores: El primero es la estructura microscópica y macroscópica de la dentina en los dos tipos de dientes y el segundo es la interface adhesiva. La adhesión a dentina se logra mediante la hibridación de la dentina que consiste en la infiltración de monómeros resinosos en las fibrillas colágenas de la estructura de la dentina que al polimerizarse las envuelven y forman la capa hibrida o zona de interdifusion resinosa ; otro mecanismo mediante el cual se logra la adhesión es la formación de tags y microtags que consiste en la penetración de los monómeros resinosos hacia los túbulos dentinales formando prolongaciones y anastomosis en la dentina. ${ }^{(1)}$ De acuerdo a los resultados obtenidos en este estudio se demostró que aunque se encontró algún grado de adhesión en los dientes con dentinogenesis imperfecta tipo II, son mucho menores a los valores de microtensión encontrados en dientes en condiciones de normalidad lo cual nos permite confirmar lo sugerido por Gallusi y cols. ${ }^{(9)}$ Los sistemas de adhesión actuales se han desarrollado y estudiado para ser utilizados en dentina normal, esta demostrado que las alteraciones se presentan en la capa hibrida; lo que permite identificar en donde pueden estar las fallas en la adhesión. Dicho esto, se puede inferir que al existir alteración en la conformación estructural de la dentina y disminución en la mineralización de dientes con dentinogenesis imperfecta, los patrones y mecanismos mediante los cuales se llega a la adhesión en dentina se encuentran alterados.

Según los resultados del presente estudio, se debe tener en cuenta que aunque existen diferencias en los valores de fuerzas de adhesión en dientes con dentinogenesis imperfecta con respecto a los dientes sanos, las restauraciones adhesivas no deben ser descartadas como alternativas de tratamiento, ya que se pudo observar que existe algún grado de adhesión, el cual puede ser comparado con valores de fuerzas de adhesión reportados en algunos estudios sobre materiales utilizados y en dientes sanos después de haber recibido algún tipo de tratamiento. ${ }^{(10)}$ Existen reportes de caso en los cuales se han utilizado restauraciones adhesivas como tratamiento presentando éxito clínico. ${ }^{(11)}$

Con el fin de mejorar las técnicas adhesivas en dientes con dentinogenesis imperfecta se sugiere combinar la adhesión con la retención mecánica ya que se ha demostrado que al realizar restauraciones tipo coronas completas se encuentra mayor retención cuando se realizan preparaciones con diseños que incorporan rieleras y elementos de retención auxiliares.

Resulta necesario seguir realizando estudios que permitan comparar el grado de adhesión de entre diferentes materiales, aplicar modificaciones en los protocolos que se utilizan para los agentes adhesivos y además pensar en nuevas alternativas en la conformación de cavidades y preparaciones. De esta manera 
se puede llegar a determinar cuáles son las mejores alternativas para rehabilitar pacientes con dentinogenesis imperfecta. 


\section{BIBLIOGRAFÍA}

1. Dourado Loguercio A, Reis A. Sistemas Adhesivos. Rev Oper Dent y Biomater Dent y Biomater. 2006;1(2):13-28.

2. Garrofé A, Martucci D, Picca M. Adhesión a tejidos dentarios. Rev Fac Odon UBA. 2014; 29 (67):5-13.

3. Seow WK. Developmental defects of enamel and dentine: Challenges for basic science research and clinical management. Aust Dent J. 2014;59(SUPPL. 1):143-54.

4. Hart PS, Hart TC. Disorders of human dentin. Cells Tissues Organs. 2007;186(1):70-7.

5. Alejandra P, Elorza B, Vyver V Der. de una resina compuesta al esmalte dental 1 INFLUENCE OF POST-BLEACHING TIME ON A COMPOSITE RESIN BOND STRENGTH TO ENAMEL 1 The effects of bleaching agents on both dental tissues breaking down into oxygen and water with heat presence of catalysts , trigge. Rev Fac Odontol Univ Antioq. 2013;25(1):92-116.

6. Dominguez J, Dominguez JA, Luque I, Horning D, Gomes JC, Gomes OMM. Evaluación De La Resistencia De Unión De Adhesivo Autograbador a Base De Silorano. Rev Colomb Investig en Odontol. 2011;2(4):7-14.

7. Liliana M, Enciso P, Liliana M, Enciso P. Microtensión en Tejidos Dentarios Duros : Desarrollo Tecnológico Microtensión en Tejidos Dentarios Duros : Desarrollo Tecnológico. Fac Odontol Dep Salud Oral Bogotá Univesidad Nac Colomb. 2015;19-30.

8. De Munck J, Mine A, Poitevin A, Van Ende A, Cardoso MV, Van Landuyt KL, et al. Metaanalytical review of parameters involved in dentin bonding. J Dent Res. 2012;91(4):351-7.

9. Gallusi G, Libonati A, Campanella V. SEM-morphology in dentinogenesis imperfecta type II: microscopic anatomy and efficacy of a dentine bonding system. Eur J Paediatr Dent. 2006;7(1):9-17.

10. Lozada MP. Sistemas adhesivos autograbadores , resistencia de unión y nanofiltración: Una revisión. Rev facultad Odontologia UDEA . 2012;24:133-50.

11. Gama FJR, Corrêa IS, Valerio CS, Ferreira E de F, Manzi FR. Dentinogenesis imperfecta type II: A case report with 17 years of follow-up. Imaging Sci Dent. 2017;47(2):129-33. 


\section{ANEXOS}

figura 1. Prueba de normalidad

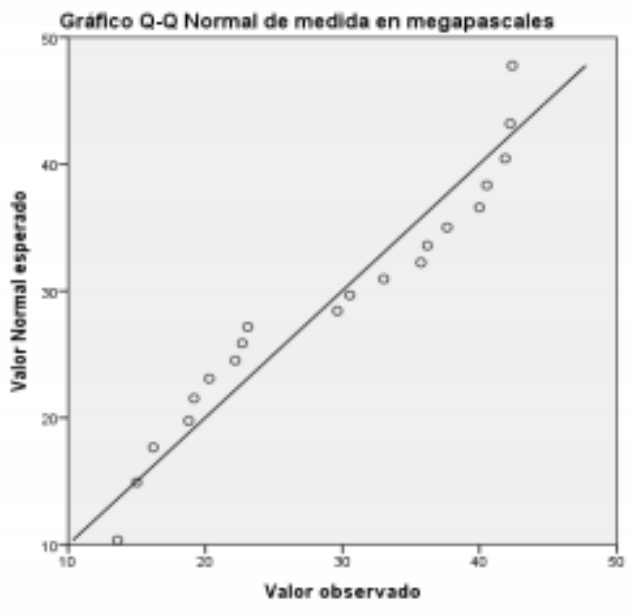

Figura 2. Comparación valores de microtensión

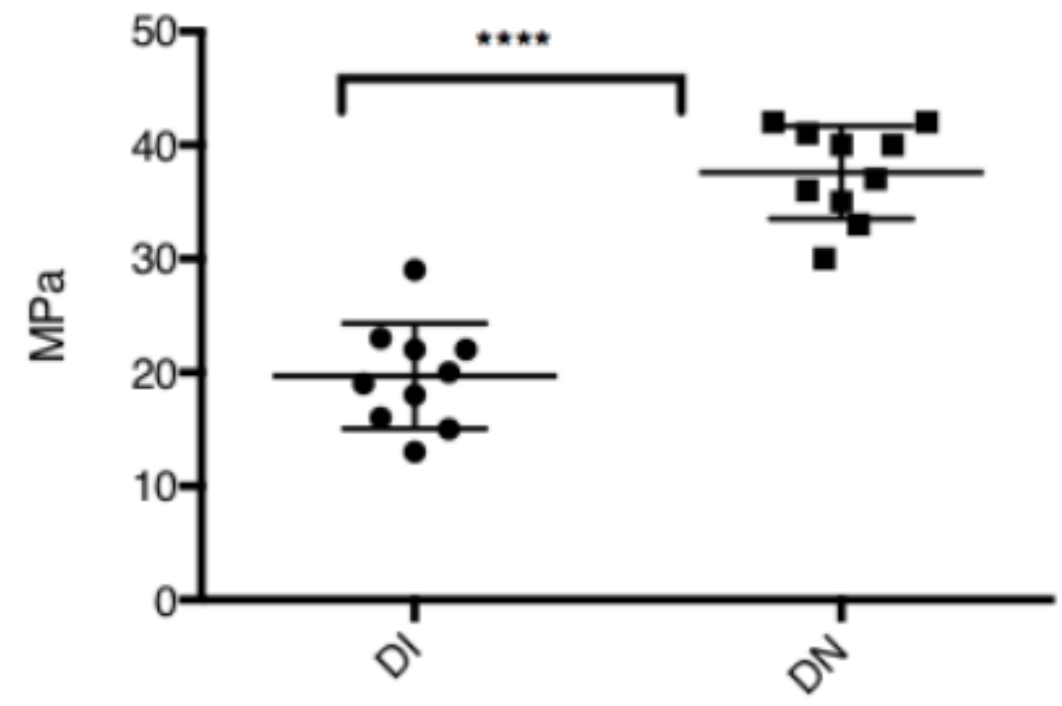

****(estadisticamente significativo) $(p<.0 .005)$.

MPa: Megapascales, DI: Dentinogenesis imperfecta, DN: Dentina normal. 
tabla 1. Frecuencia de acuerdo al tipo de falla.

\begin{tabular}{lll}
\hline TIPO DE FALLA & Frecuencia & Porcentaje \\
Adhesiva & 9 & $45 \%$ \\
Cohesiva & 5 & $25 \%$ \\
Mixta & 6 & $30 \%$ \\
Total & 20 & $100 \%$ \\
\hline
\end{tabular}

tabla 2. Comparación tipo de diente con tipo de falla

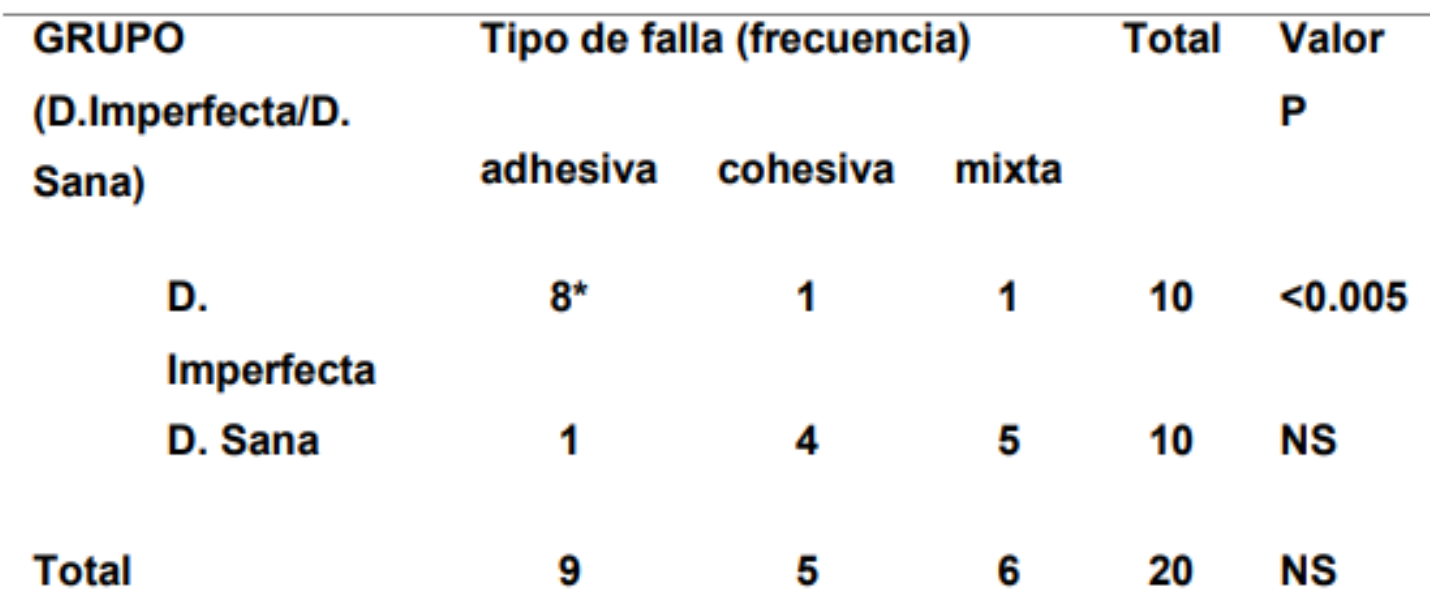

NS=No significativo; *Diferencia estadísticamente significativa 